\title{
Integrating Machine Learning with Liquid-Phase TEM Imaging to Study Nanoscale Crystallization and Macromolecular Heterogeneity
}

\author{
Chang Qian ${ }^{1}$, Lehan Yao ${ }^{1}$, Chang Liu ${ }^{1}$, John W. Smith ${ }^{1}$ and Qian Chen ${ }^{1}$ \\ ${ }^{1}$ Department of Materials Science and Engineering, University of Illinois at Urbana-Champaign.
}

Machine learning (ML) has found great opportunities in electron microscopy related applications such as atomic structure analysis on scanning transmission electron microscopy (STEM) images [1], dose efficient acquisition by compressive sensing [2], and shape classification on transmission electron microscopy (TEM) images [3]. Its integration with liquid-phase TEM can bring technical advancements at all levels from smart imaging, smart segmentation of low signal-to-noise ratio (SNR) features, to smart recognition of structural and energetic fingerprints in a dynamic process. For example, we have previously customized an image segmentation workflow based on a U-Net convolutional neural network (CNN) for inorganic nanoparticles imaged by liquid-phase TEM [4], where a probability map of a pixel belonging to the features is extracted as outputs to generate the shape contours of the features. Various parameters were quantified using this workflow, such as the interaction maps, anisotropic etching rates, and self-assembly kinetic laws of nanoparticles.

Here we build upon this earlier effort of ours to further integrate ML and liquid-phase TEM in two unexplored aspects. The first is on the crystallization of nanoparticle building blocks in solution. While our previous studies focus on the initial nucleation [5] and expansion of crystal surfaces described by a capillary wave theory $[6,7]$, in this work we study the translational and rotational trajectories of the nanoparticles as they sit at the lattice sites. This single-particle level dynamics can relate to a diversity of reconfigurable properties (e.g., structural and mechanical) at the crystal level. To do so, for single-layered crystals, U-Net was used to segment individual nanoparticles (with watershed applied if needed) to track the centroids and orientations of the nanoparticles (Fig. 1a) in each TEM snapshot. Continuous trajectories were then obtained throughout the liquid-phase TEM movie (Fig. 1a), which are both highly localized due to the confined environment surrounded by nearest neighbors. For thick assembled structures, two output channels in U-Net were designed to distinguish the overlaying nanoparticles when multiple layers are present and projected on top of each other in TEM (Fig. 1b). The second is on recognizing macromolecular heterogeneity in liquid-phase TEM videos. These macromolecular systems are highly beam-sensitive. They can only be imaged at low dose rates and thus exhibit low SNR. In this case, ML-based denoising methods can enhance the contrast of the TEM images, facilitating the recognition of structural heterogeneity at the nanoscale. Here $\mathrm{CNN}$ - based segmentation alone does not work efficiently due to the difficulty to generate simulated liquid-phase TEM images as the training dataset. We see that the above two efforts in integrating ML with liquid-phase TEM lay the foundation for understanding complex phase behaviors or self-organization pathways at the nanoscale, and the fundamental structural heterogeneity in soft nanomaterials. 
References:

[1] M Ziatdinov et al, ACS Nano 11 (2017), p. 12742.

[2] A Stevens et al, Appl Phys Lett 113 (2018), p. 033104.

[3] X Wang et al, JACS Au 1 (2021), p. 316.

[4] L Yao et al, ACS Cent. Sci. 6 (2020), p. 1421.

[5] Z Ou et al, Nat. Mater. 19 (2020), p. 450.

[6] Z Ou et al, Nat. Commun. 11 (2020), p. 4555.

[7] C Liu et al, J. Am. Chem. Soc. 142 (2020), p. 11669.

[8] The authors acknowledge support from the National Science Foundation under grant no. 1752517.

\section{a TEM movie}

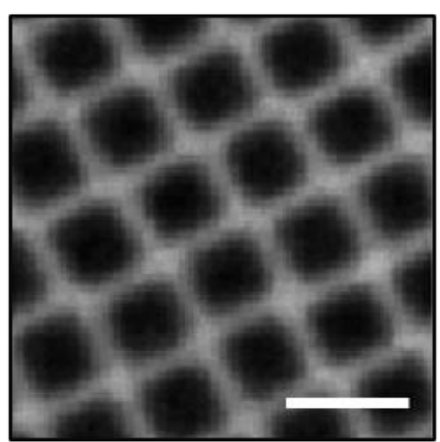

b
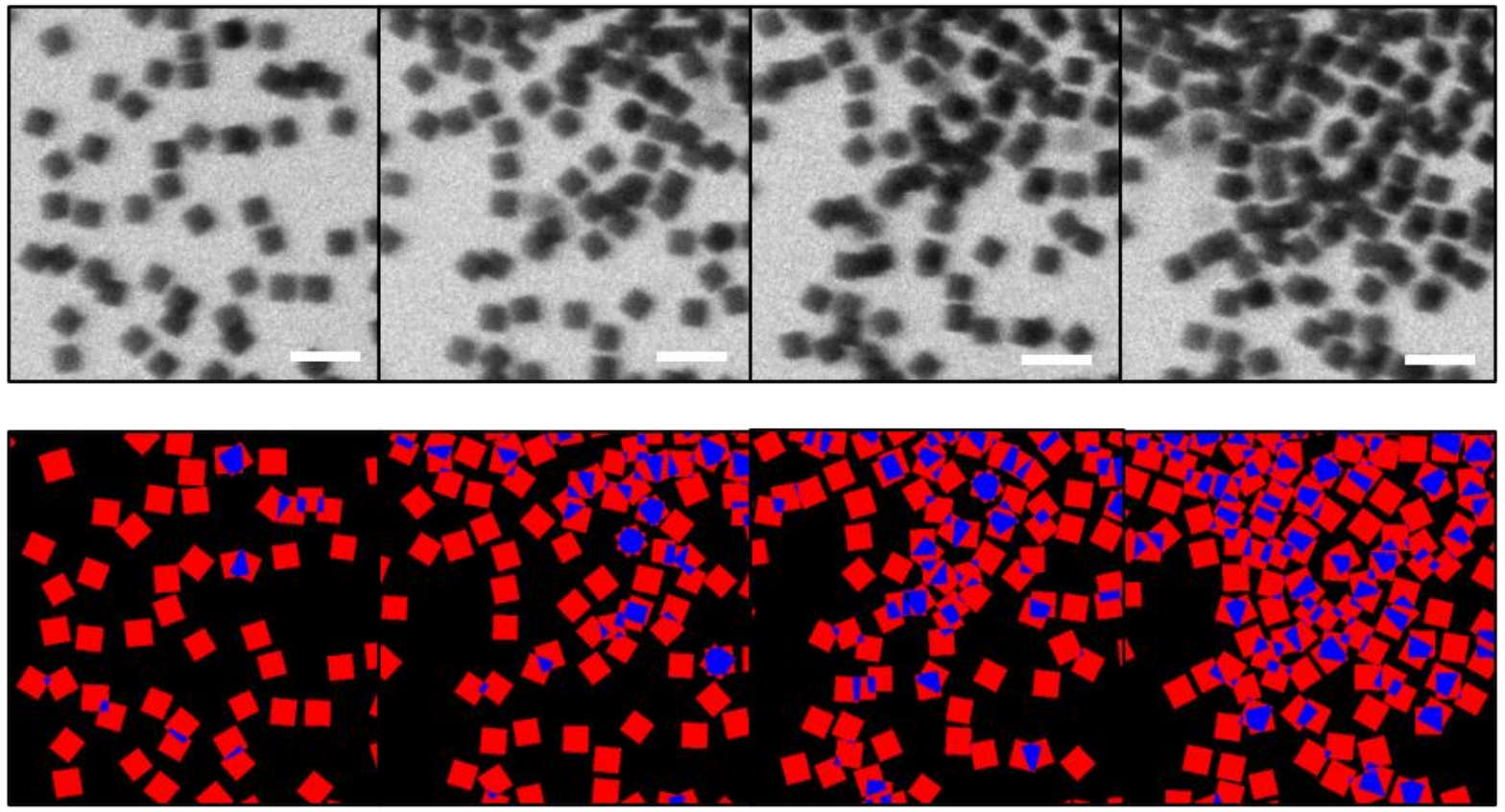

Figure 1: Demonstrations of ML to track the centroid and orientations of individual nanocubes in a crystal imaged by liquid-phase TEM (a) and to assign overlaying nanoparticles to different layers (b). Scale bars: $100 \mathrm{~nm}$.
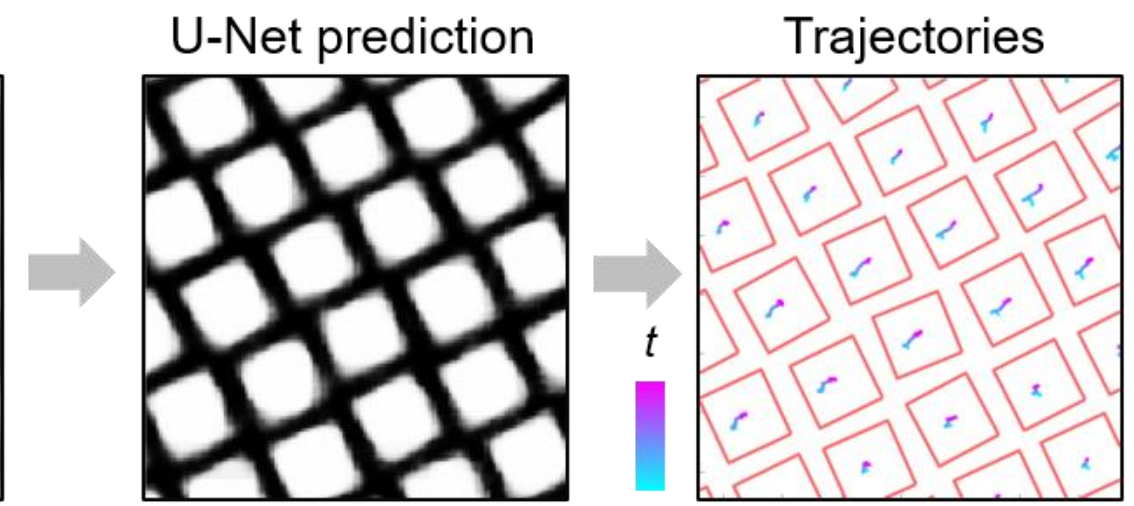

$$
\text { 西 }
$$

